\title{
Stereoselective Synthesis of $\alpha$-Silylamines by the Direct Addition of Silyl Anions to Activated Imines
}

\author{
David M. Ballweg, Rebecca C. Miller, Danielle L. Gray and Karl A. Scheidt* \\ Supporting Information
}

General Information. All reactions were carried out under a nitrogen atmosphere in flame-dried glassware with magnetic stirring. THF and $\mathrm{CH}_{2} \mathrm{Cl}_{2}$, were purified by passage through a bed of activated alumina. ${ }^{1}$ Reagents were purified prior to use unless otherwise stated following the guidelines of Perrin and Armarego. ${ }^{2}$ Purification of reaction products were carried out by flash chromatography using EM Reagent silica gel 60 (230-400 mesh). Analytical thin layer chromatography was performed on EM Reagent $0.25 \mathrm{~mm}$ silica gel $60-\mathrm{F}$ plates. Visualization was accomplished with UV light and staining with ceric ammonium molybdate (CAM). Infrared spectra were recorded on a Perkin Elmer 1600 series FT-IR spectrometer. ${ }^{1} \mathrm{H}-\mathrm{NMR}$ spectra were recorded on a Mercury $400(400 \mathrm{MHz}$ ) spectrometer and are reported in ppm using solvent as an internal standard $\left(\mathrm{CDCl}_{3}\right.$ at $\left.7.26 \mathrm{ppm}\right)$. Data are reported as $($ ap $=$ apparent, $\mathrm{s}=$ singlet, $\mathrm{d}=$ doublet, $\mathrm{t}=$ triplet, $\mathrm{m}=$ multiplet, $\mathrm{b}=$ broad; coupling constant( $\mathrm{s})$ in $\mathrm{Hz}$. Protondecoupled ${ }^{13} \mathrm{C}-\mathrm{NMR}$ spectra were recorded on a Mercury $400(100 \mathrm{MHz})$ spectrometer and are reported in ppm using solvent as an internal standard $\left(\mathrm{CDCl}_{3}\right.$ at $\left.77.23 \mathrm{ppm}\right)$. Proton-decoupled ${ }^{31} \mathrm{P}-\mathrm{NMR}$ spectra were recorded on a Mercury $400(162 \mathrm{MHz})$ spectrometer and are reported in ppm and were referenced to $\mathrm{H}_{3} \mathrm{PO}_{4}$ as an external standard. Proton-decoupled ${ }^{19} \mathrm{~F}-\mathrm{NMR}$ spectra were recorded on a Mercury 400 (376 $\mathrm{MHz}$ ) spectrometer and are reported in ppm and were referenced to $\mathrm{CFCl}_{3}$ in $\mathrm{CDCl}_{3}$ as an external standard. Proton-decoupled ${ }^{29} \mathrm{Si}-\mathrm{NMR}$ spectra were recorded on a Varian Inova 500 (99 Mhz) or Varian Inova $400(80 \mathrm{Mhz})$ spectrometer and are reported in ppm and were referenced to tetramethylsilane in $\mathrm{CDCl}_{3}$ as an external standard. Mass spectra data were obtained on a Varian 1200 quadrupole (MALDI), or Micromass Quattro II triple quadrupole HPLC/MS/MS (ESI).

Crystallographic Information. Single-crystal X-ray diffraction data were collected with the use of graphite-monochromatized Mo K $\alpha$ radiation $(\lambda=0.71073 \AA)$ at $153 \mathrm{~K}$ on a Bruker Smart-1000 CCD diffractometer with the program SMART. ${ }^{3}$ The crystal-to-detector distance was $5.023 \mathrm{~cm}$. Data was collected by a scan of $0.3^{\circ}$ in $\omega$ in groups of 606 frames at settings of $0^{\circ}, 120^{\circ}$, and $240^{\circ}$. Crystal decay was monitored by re-collecting 50 initial frames at the end of data collection. Cell refinement and data reduction were carried out with the use of the program SAINT, and face-indexed absorption corrections were performed numerically with the use of the program XPREP. Then the program SADABS was employed to make incident beam and decay correction. The structure was solved by means of the direct methods program SHELXS of the SHELXTL-PC suite of programs. ${ }^{4}$ All the non-hydrogen atoms were located from the trial structure and then refined anisotropically with SHELXTL using the full-matrix

(1) Pangborn, A. B.; Giardello, M. A.; Grubbs, R. H.; Rosen, R. K.; Timmers, F. J. Organometal. 1996, 15, 1518-1520.

(2) Perrin, D. D. and Armarego, W. L. Purification of Laboratory Chemicals; 3rd Ed., Pergamon Press, Oxford. 1988.

(3) SMART Version 5.054 Data Collection and SAINT-Plus Version 6.45 Data Processing Software for the SMART System; Bruker Analytical X-Ray Instruments, Inc.: Madison, WI, 2003.

(4) Sheldrick, G. M. SHELXTL PC Version 6.10 An Integrated System for Solving, refining, and Displaying Crystal Structure from Diffraction Data; Siemens Analytical X-Ray instruments, Inc.: Madison, WI, 1994. 
least-squares procedure. The crystallographic data can be obtained free of charge via the internet (www.ccdc.cam.ac.uk/conts/retrieving.html), by mail at Cambridge Crystallographic Data Centre, 12, Union Road, Cambridge CB21EZ, UK, by fax at (+44) 1223-336-033, or e-mail (deposit@ccdc.cam.ac.uk).

\section{General procedure for the synthesis of $\boldsymbol{N}$-diphenylphosphinoyl imines:}

All imines were prepared using a modification to the procedure of Jennings and coworkers. ${ }^{5}$ As a sample procedure: To a $50 \mathrm{~mL}$ Schlenk flask was added $P, P$-diphenylphosphinic amide $(0.90 \mathrm{~g}, 4.38 \mathrm{mmol}), \mathrm{m}$ tolualdehyde $(0.52 \mathrm{~mL}, 4.38 \mathrm{mmol}), \mathrm{NEt}_{3}(2.14 \mathrm{~mL}, 15.3 \mathrm{mmol})$, and $\mathrm{CH}_{2} \mathrm{Cl}_{2}(30 \mathrm{~mL})$. The mixture was then cooled to $0{ }^{\circ} \mathrm{C}$. To this mixture was added a solution of $\mathrm{TiCl}_{4}(2.19 \mathrm{mmol})$ in $\mathrm{CH}_{2} \mathrm{Cl}_{2}(2 \mathrm{~mL})$ dropwise. After the addition was complete, typically 5 minutes, the mixture was stirred for 1 hour at 0 ${ }^{\circ} \mathrm{C}$, then 1 hour at room temperature. The reaction mixture was then filtered through Celite to remove $\mathrm{TiO}_{2}$ and washed with $\mathrm{CH}_{2} \mathrm{Cl}_{2}$. Rotary evaporation of the filtrate gave a solid mixture of imine and triethylamine hydrochloride. The residue was purified by flash column chromatography $(70 \%$ EtOAc/hexane) to afford pure imine.

The $t$-butanesulfinimines used in this study were prepared using methods developed by Ellman and coworkers. ${ }^{6} \mathrm{~N}$-(Diethoxyphosphoryl)aldimine was synthesized using the method reported by Barrow and coworkers. $^{7}$

\section{General procedure for protected $\alpha$-silylamines:}

To a $25 \mathrm{~mL}$ Schlenk flask was added 1 equivalent of imine (approx. $100 \mathrm{mg}$ ), and THF (3 mL), and the mixture was cooled to $-78{ }^{\circ} \mathrm{C}$. To this mixture was added 3 equivalents of silyl lithium (approx. $1 \mathrm{M}$ in THF). After stirring at $-78{ }^{\circ} \mathrm{C}$ for 20 minutes, the reaction was quenched with $5 \%$ acetic acid in methanol $(10 \mathrm{~mL})$ at $-78{ }^{\circ} \mathrm{C}$. The mixture was taken up with ethyl acetate $(20 \mathrm{~mL})$, washed with water $(1 \times 10$ $\mathrm{mL})$, brine $(2 \times 10 \mathrm{~mL})$. The organic layer was dried over anhydrous sodium sulfate, filtered, and concentrated in vacuo. The unpurified reaction mixture was separated using flash chromatography (EtOAc/hexanes) to yield pure product.

(5) Jenning, W. B.; Lovely, C.J.; Tetrahedron. 1991, 47, 5561-5568.

(6) Ellman, J. A.; Cogan, D. A.; Liu, G. J. Am. Chem. Soc. 1997, 119, 9913-9914. 


\section{Selected Characterization Data:}

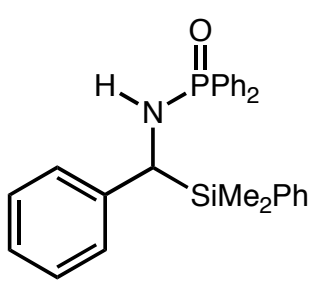

$\boldsymbol{P}, \boldsymbol{P}$-diphenyl- $\boldsymbol{N}$-(1-dimethylphenylsilylphenylmethylene)-phosphinic amide (7): Purified with $80 \%$ ethyl acetate/hexanes to yield $104.6 \mathrm{mg}$ ( $88 \%$ yield) of colorless crystals. Mp: $128-131{ }^{\circ} \mathrm{C} ; \mathrm{R}_{f}=0.50$ (80/20 ethyl acetate/hexanes); IR (film) 3396, 3326, 3060, 1436, 1192, 1111, 723, $689 \mathrm{~cm}^{-1}$; ${ }^{1} \mathrm{H} \mathrm{NMR}\left(400 \mathrm{MHz}, \mathrm{CDCl}_{3}\right) \delta$ 7.68$7.55(\mathrm{~m}, 4 \mathrm{H}), 7.48-7.28(\mathrm{~m}, 9 \mathrm{H}), 7.26-7.06(\mathrm{~m}, 5 \mathrm{H}), 6.79($ ap d, $J=7.3 \mathrm{~Hz}, 2 \mathrm{H}), 3.94$ $(\mathrm{dd}, J=11.0,11.0 \mathrm{~Hz}, 1 \mathrm{H}), 3.20(\mathrm{dd}, J=9.2,9.2 \mathrm{~Hz}, 1 \mathrm{H}), 0.439$ (s, 3H), 0.217 (s, $3 \mathrm{H}) ;{ }^{13} \mathrm{C}$ NMR $\left(100 \mathrm{MHz}, \mathrm{CDCl}_{3}\right) \delta 142.5,134.8,132.7$ (d, $\left.J=10.1 \mathrm{~Hz}\right), 131.9$ (d, $\left.J=9.2 \mathrm{~Hz}\right), 131.8$ (d, $J=2.8 \mathrm{~Hz}), 131.7(\mathrm{~d}, J=2.8 \mathrm{~Hz}), 130.0,128.6,128.4,128.2,128.1,128.1,128.0,126.4,125.6,47.2(\mathrm{~d}, J$ $=3.7 \mathrm{~Hz}),-4.5,-5.0 ;{ }^{31} \mathrm{P} \mathrm{NMR}\left(162 \mathrm{MHz}, \mathrm{CDCl}_{3}\right) \delta 25.6 ;{ }^{29} \mathrm{Si} \mathrm{NMR}\left(99 \mathrm{MHz}, \mathrm{CDCl}_{3}\right) \delta-0.64 ; \mathrm{LRMS}$ (MALDI-TOF): Mass calculated for $\mathrm{C}_{27} \mathrm{H}_{28} \mathrm{NOPSi}[\mathrm{M}+\mathrm{H}]+:$ 442.6. Found 442.5.

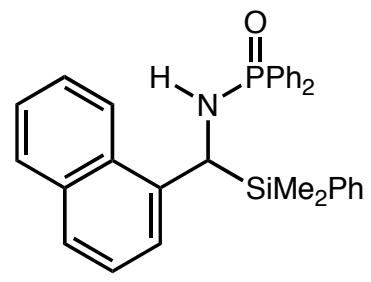

$P, P$-diphenyl- $N$-(1-dimethylphenylsilyl-1-naphthylmethylene)-phosphinic amide (9): Purified with $80 \%$ ethyl acetate/hexanes to yield $87 \mathrm{mg}$ (64\% yield) of colorless crystals. Mp: $117-120{ }^{\circ} \mathrm{C} ; \mathrm{R}_{f}=0.50$ (80/20 ethyl acetate/hexanes); IR (film) 3392, 1647, 1561, 1188, $1112 \mathrm{~cm}^{-1} ;{ }^{1} \mathrm{H}$ NMR $\left(400 \mathrm{MHz}, \mathrm{CDCl}_{3}\right)$ 7.77$7.72(\mathrm{~m}, 1 \mathrm{H}), 7.67-7.49(\mathrm{~m}, 5 \mathrm{H}), 7.47-7.25(\mathrm{~m}, 11 \mathrm{H}), 7.24-7.12(\mathrm{~m}, 3 \mathrm{H}), 7.08-$ $7.02(\mathrm{~m}, 2 \mathrm{H}), 4.83(\mathrm{dd}, J=10.4,10.4 \mathrm{~Hz}, 1 \mathrm{H}), 3.36(\mathrm{dd}, J=8.5,9.2 \mathrm{~Hz}, 1 \mathrm{H})$, 0.475 (s, 3h), 0.039 (s, 3H); ${ }^{13} \mathrm{C}$ NMR (100 MHz, $\left.\mathrm{CDCl}_{3}\right) \delta 140.4$ (d, J=2.1 Hz), 139.4, 134.9, 134.7, $133.4(\mathrm{~d}, J=3.1 \mathrm{~Hz}), 132.5(\mathrm{~d}, J=10.1 \mathrm{~Hz}), 132.4,131.9(\mathrm{~d}, J=9.2 \mathrm{~Hz}), 131.7(\mathrm{~d}, J=2.7 \mathrm{~Hz}), 131.5(\mathrm{~d}$, $J=2.7 \mathrm{~Hz}), 131.1(\mathrm{~d}, J=3.3 \mathrm{~Hz}), 130.3(\mathrm{~d}, J=2.0 \mathrm{~Hz}), 130.0,128.6,128.4(\mathrm{~d}, J=11.9 \mathrm{~Hz}), 128.1$, 128.0, 126.1, 125.3, 125.3, 124.0, 122.9, 41.8, -3.9, $-4.8(\mathrm{~m}) ;{ }^{31} \mathrm{P}$ NMR $\left(162 \mathrm{MHz}, \mathrm{CDCl}_{3}\right) \delta 25.4$; LRMS (MALDI-TOF): Mass calculated for $\mathrm{C}_{31} \mathrm{H}_{30} \mathrm{NOPSi}[\mathrm{M}+\mathrm{H}]+$ : 492.6. Found 493.0.

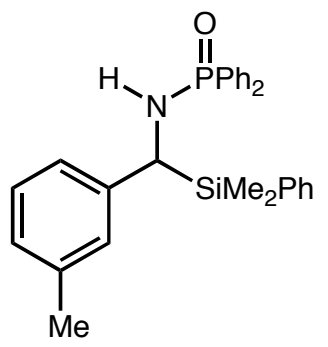

$\boldsymbol{P}, \boldsymbol{P}$-diphenyl- $\boldsymbol{N}$-(1-dimethylphenylsilyl-3-methylphenylmethylene)-phosphinic amide (11): Purified with 80\% ethyl acetate/hexanes to yield $105 \mathrm{mg}$ (74\% yield) of colorless crystals. Mp: $129-130{ }^{\circ} \mathrm{C} ; \mathrm{R}_{f}=0.50$ (80/20 ethyl acetate/hexanes); IR (film) 3451, 3223, 3048, 1429, 1187, 1113, $691 \mathrm{~cm}^{-1} ;{ }^{1} \mathrm{H}$ NMR (400 $\left.\mathrm{MHz}, \mathrm{CDCl}_{3}\right)$ $\delta$ 7.68-7.52 (m, 4H), 7.48-7.14 (m, 11H), 7.04 (ap t, $J=7.3 \mathrm{~Hz}, 1 \mathrm{H}), 6.89$ (ap d, $J$ $=7.2 \mathrm{~Hz}, 1 \mathrm{H}), 6.63($ ap d $, J=7.2,1 \mathrm{H}), 6.51(\mathrm{~s}, 1 \mathrm{H}), 3.90(\mathrm{dd}, J=11.0,11 \mathrm{~Hz}, 1 \mathrm{H})$, $3.19(\mathrm{dd}, J=9.8,9.5 \mathrm{~Hz}, 1 \mathrm{H}), 2.19(\mathrm{~s}, 3 \mathrm{H}), 0.429(\mathrm{~s}, 3 \mathrm{H}), 0.217(\mathrm{~s}, 3 \mathrm{H}) ;{ }^{13} \mathrm{C} \mathrm{NMR}$ $\left(100 \mathrm{MHz} \mathrm{CDCl}_{3}\right) \delta 142.3(\mathrm{~d}, J=1.8 \mathrm{~Hz}), 137.2,135.0,134.8,132.7(\mathrm{~d}, J=9.1$ $\mathrm{Hz}), 131.9,131.8,131.7(\mathrm{~d}, J=2.8 \mathrm{~Hz}), 131.6(\mathrm{~d}, J=2.8 \mathrm{~Hz}), 129.9,128.4(\mathrm{~d}, J=12.7 \mathrm{~Hz}), 128.2,128.0$, 128.0, 127.8, 127.3, 126.3, 123.5, $47.1(\mathrm{~d}, J=3.6 \mathrm{~Hz}), 21.6,-4.5,-5.0(\mathrm{~d}, J=22.7 \mathrm{~Hz}) ;{ }^{31} \mathrm{P}$ NMR $(162$ $\left.\mathrm{MHz}, \mathrm{CDCl}_{3}\right) \delta 25.6 ;{ }^{29} \mathrm{Si} \mathrm{NMR}\left(99 \mathrm{MHz}, \mathrm{CDCl}_{3}\right) \delta-0.90(\mathrm{~d}, J=10.7 \mathrm{~Hz})$; LRMS (MALDI-TOF): Mass calculated for $\mathrm{C}_{28} \mathrm{H}_{30} \mathrm{NOPSi}[\mathrm{M}+\mathrm{H}]+$ : 456.6. Found 457.1.

Crystal data for 11: $\mathrm{C}_{28} \mathrm{H}_{30} \mathrm{NOPSi}, \mathrm{M}_{\mathrm{r}}=455.59$, crystal dimensions $=0.266 \times 0.188 \times 0.048 \mathrm{~mm}$, triclinic, space group P1(bar), $a=9.7494(8), b=12.3191(11), c=12.4391(11) \AA, \alpha=101.7890(10), \beta=$ 111.8390(10), $\gamma=107.5441(10)^{\circ}, \mathrm{V}=1234.85(19) \AA^{3}, \mathrm{Z}=2, \rho_{\text {calcd }}=1.225 \mathrm{~g} \mathrm{~cm}^{-3}, 2 \theta_{\mu \alpha \xi}=57.264$, number of measured reflections $=11425$, number of unique reflections $=5762$, number of parameters $=293, R(F)$ $=\Sigma\left\|F_{o}|-| F_{c}\right\| / \Sigma\left|F_{o}\right|$ for $F_{o}{ }^{2}>2 \sigma\left(F_{o}{ }^{2}\right) . R_{w}\left(F^{2}\right)=\left\{\Sigma\left[\left(F_{o}{ }^{2}-F_{c}{ }^{2}\right)^{2}\right] / \Sigma w \mathrm{~F}_{o}{ }^{4}\right\}^{1 / 2}$ for all data, $w^{-1}=\sigma^{2}\left(F_{o}{ }^{2}\right)+$ $\left(0.077 F_{o}{ }^{2}\right)^{2}$ for $F_{o}{ }^{2} \geq 0$ and $w^{-1}=\sigma^{2}\left(F_{o}{ }^{2}\right)$ for $F_{o}{ }^{2}<0, \mathrm{R}_{1}(F)=0.0492, \mathrm{wR}\left(F^{2}\right)=0.1308$, exposure time $=$ $15 \mathrm{~s} /$ frame. The hydrogen atom positions were fixed geometrically at calculated distances and allowed to ride on the parent atoms. Reference number for the Cambridge data base: CCDC 257700. 


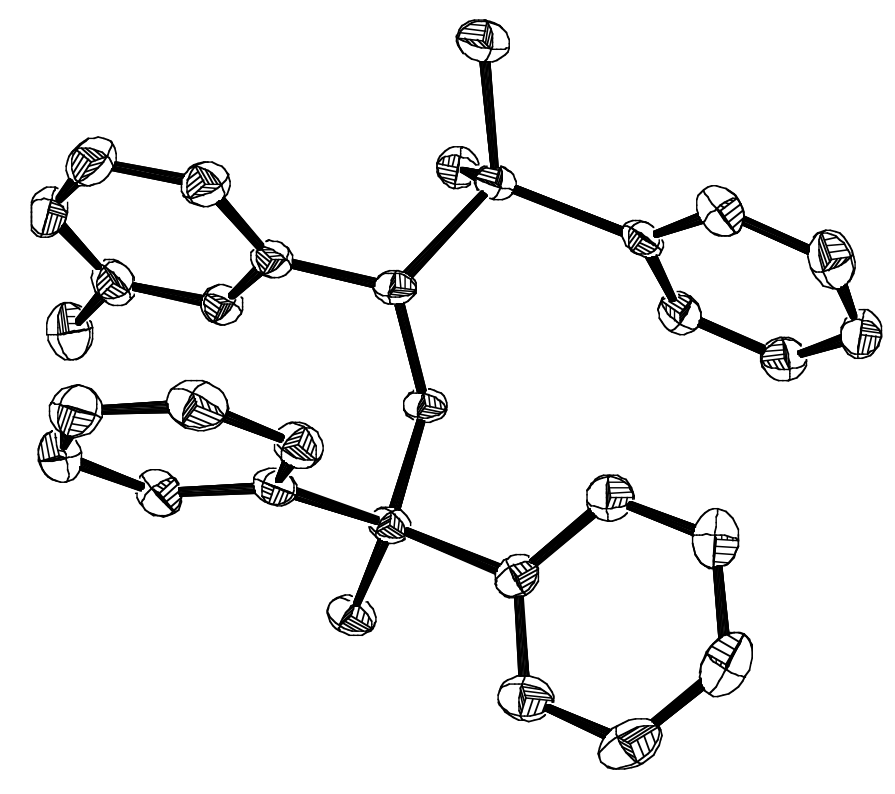

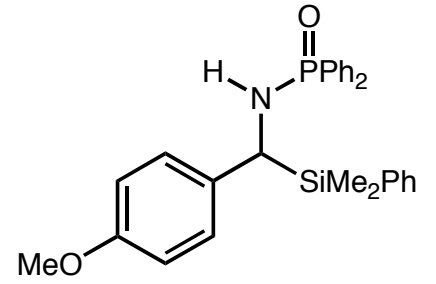

$\boldsymbol{P}, \boldsymbol{P}$-diphenyl- $\boldsymbol{N}$-(1-dimethylphenylsilyl-4-methoxyphenylmethylene)phosphinic amide (14): Purified with $80 \%$ ethyl acetate/hexanes to yield 111 mg (86\% yield) of colorless crystals. Mp: $98-100{ }^{\circ} \mathrm{C} ; \mathrm{R}_{f}=0.52$ (80/20 ethyl acetate/hexanes); IR (film) 3215, 3043, 1507, 1435, 1245, 1173, 1104, $698 \mathrm{~cm}^{-}$ ${ }^{1} ;{ }^{1} \mathrm{H}$ NMR (400 MHz, $\left.\mathrm{CDCl}_{3}\right)$ 7.67-7.57 (m, 4H), 7.45-7.19 (m, 11H), $6.71(\mathrm{~s}$, $4 \mathrm{H}), 3.87(\mathrm{dd}, J=11.0,10.4 \mathrm{~Hz}, 1 \mathrm{H}), 3.76(\mathrm{~s}, 3 \mathrm{H}), 3.18(\mathrm{dd}, J=9.8,9.2 \mathrm{~Hz}$, $\mathrm{Hz}), 132.6(\mathrm{~d}, J=9.2 \mathrm{~Hz}), 131.9(\mathrm{~d}, J=9.2 \mathrm{~Hz}), 131.7(\mathrm{~d}, J=2.7 \mathrm{~Hz}), 131.6(\mathrm{~d}, J=2.7 \mathrm{~Hz}), 129.9,128.4$ $(\mathrm{d}, J=11.9 \mathrm{~Hz}), 128.1(\mathrm{~d}, J=12.8 \mathrm{~Hz}), 128.0,127.4,113.4,55.4,46.4(\mathrm{~d}, J=4.6 \mathrm{~Hz}),-4.5,-5.1(\mathrm{~d}, J=$ $1.8 \mathrm{~Hz}) ;{ }^{31} \mathrm{P}$ NMR $\left(162 \mathrm{MHz}, \mathrm{CDCl}_{3}\right) \delta 25.4$; LRMS (MALDI-TOF): Mass (M+Na) calculated for $\mathrm{C}_{28} \mathrm{H}_{30} \mathrm{NO}_{2} \mathrm{PSi}[\mathrm{M}+\mathrm{Na}]+$ : 494.6. Found 495.3.

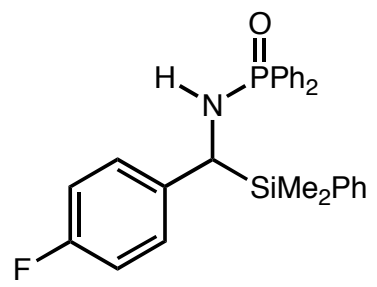

$\boldsymbol{P}, \boldsymbol{P}$-diphenyl- $\boldsymbol{N}$-(1-dimethylphenylsilyl-4-fluorophenylmethylene)phosphinic amide (15): Purified with $80 \%$ ethyl acetate/hexanes to yield 114 mg (77\% yield) of colorless crystals. Mp: $153-155{ }^{\circ} \mathrm{C} ; \mathrm{R}_{f}=0.53(80 / 20$ ethyl acetate/hexanes); IR (film) 3362, 3208, 1185, 1119, 1110, $699 \mathrm{~cm}^{-1} ;{ }^{1} \mathrm{H}$ NMR $\left(400 \mathrm{MHz}, \mathrm{CDCl}_{3}\right)$ 7.66-7.54 (m, 4H), 7.49-7.18 (m, 11H), 6.88-6.70 (m, 4H), $3.92(\mathrm{dd}, J=10.7,10.7 \mathrm{~Hz}, 1 \mathrm{H}), 3.19(\mathrm{dd}, J=9.2,9.2 \mathrm{~Hz}, 1 \mathrm{H}), 0.431(\mathrm{~s}, 3 \mathrm{H})$, $0.222(\mathrm{~s}, 3 \mathrm{H}) ;{ }^{13} \mathrm{C}$ NMR $\left(100 \mathrm{MHz}, \mathrm{CDCl}_{3}\right) \delta 162.3,159.9,138.3,134.7,134.5$, $132.6(\mathrm{~d}, J=9.2 \mathrm{~Hz}), 131.9(\mathrm{~d}, J=9.2 \mathrm{~Hz}), 131.9,131.8(\mathrm{~d}, J=1.8 \mathrm{~Hz}), 130.1,128.5(\mathrm{~d}, J=11.9 \mathrm{~Hz})$, 128.3, 128.2, 127.8 (d, $J=7.3 \mathrm{~Hz}), 114.9,114.7,46.5(\mathrm{~d}, J=4.6 \mathrm{~Hz}),-4.6,-5.1 ;{ }^{31} \mathrm{P}$ NMR $(162 \mathrm{MHz}$, $\left.\mathrm{CDCl}_{3}\right) \delta 25.6 ;{ }^{19} \mathrm{~F}$ NMR $\left(376 \mathrm{MHz}, \mathrm{CDCl}_{3}\right) \delta$ 15.0; LRMS (MALDI-TOF): Mass calculated for $\mathrm{C}_{27} \mathrm{H}_{27}$ FNOPSi $[\mathrm{M}+\mathrm{H}]+:$ 460.2. Found 460.8 .

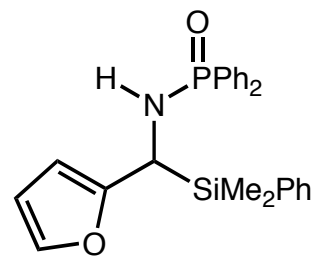

$\boldsymbol{P}, \boldsymbol{P}$-diphenyl- $\boldsymbol{N}$-(1-dimethylphenylsilyl-2-furylmethylene)-phosphinic amide (18): Purified with $80 \%$ ethyl acetate/hexanes to yield $115 \mathrm{mg}(91 \%$ yield $)$ of colorless crystals. Mp: 105-106; $\mathrm{R}_{f}=0.53$ (80/20 ethyl acetate/hexanes); IR (film) 3366, 3198, 3053, 2957, 2648, 1437, 1250, 1190, 1122, 1111, 839, 726, $696 \mathrm{~cm}^{-1} ;{ }^{1} \mathrm{H}$ NMR (400 $\left.\mathrm{MHz} \mathrm{CDCl}_{3}\right) \delta$ 7.68-7.24 (m, 16H), $6.17(\mathrm{~s}, 1 \mathrm{H}), 5.75(\mathrm{~d}, J=2.4 \mathrm{~Hz}, 1 \mathrm{H}), 4.02(\mathrm{dd}, J$ 
$=10.4,10.4 \mathrm{~Hz}, 1 \mathrm{H}), 3.12(\mathrm{dd}, J=9.8,9.8 \mathrm{~Hz}, 1 \mathrm{H}), 0.413(\mathrm{~s}, 3 \mathrm{H}), 0.350(\mathrm{~s}, 3 \mathrm{H}) ;{ }^{13} \mathrm{C} \mathrm{NMR}(100 \mathrm{MHz}$, $\left.\mathrm{CDCl}_{3}\right) \delta 155.5(\mathrm{~d}, J=2.7 \mathrm{~Hz}), 141.0,135.2,132.5,132.4,131.9,131.8,131.7(\mathrm{~d}, J=2.7 \mathrm{~Hz}), 129.9$, $128.5(\mathrm{~d}, J=11.9 \mathrm{~Hz}), 128.2(\mathrm{~d}, J=11.9 \mathrm{~Hz}), 128.0,110.5,105.7,40.4(\mathrm{~d}, J=5.4 \mathrm{~Hz}),-4.3,-4.9(\mathrm{~d}, J=$ $1.9 \mathrm{~Hz}) ;{ }^{31} \mathrm{P}$ NMR $\left(162 \mathrm{MHz}, \mathrm{CDCl}_{3}\right) \delta 25.6 ;{ }^{29} \mathrm{Si} \mathrm{NMR}\left(99 \mathrm{MHz}, \mathrm{CDCl}_{3}\right) \delta-0.123,-0.231$; LRMS (MALDI-TOF): Mass calculated for $\mathrm{C}_{25} \mathrm{H}_{26} \mathrm{NO}_{2} \mathrm{PSi}[\mathrm{M}+\mathrm{H}]+:$ : 432.5. Found 432.8.

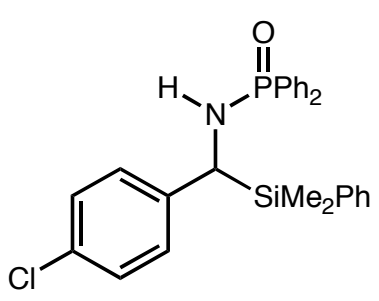

$\boldsymbol{P , P}$-diphenyl- $\boldsymbol{N}$-(1-dimethylphenylsilyl-4-chlorophenylmethylene)phosphinic amide (17): Purified with $80 \%$ ethyl acetate/hexanes to yield 102 mg $\left(83 \%\right.$ yield) of colorless crystals. Mp: 160-162; $\mathrm{R}_{f}=0.50$ (80/20 ethyl acetate/hexanes); IR (film) 3205, 3048, 1479, 1427, 1259, 1184, $1113 \mathrm{~cm}^{-1} ;{ }^{1} \mathrm{H}$ NMR (400 MHz, $\left.\mathrm{CDCl}_{3}\right)$ ठ 7.65-7.51 (m, 4H), 7.48-7.30 (m, 9H), 7.28-7.20 (m, 2H), 7.14-7.06 (m, 2H), 6.77-6.67 (m, 2H), $3.91(\mathrm{dd}, J=10,11 \mathrm{~Hz}, 1 \mathrm{H}), 3.19$ $(\mathrm{dd}, J=9.0,9.0 \mathrm{~Hz}, 1 \mathrm{H}), 0.434(\mathrm{~s}, 3 \mathrm{H}), 0.220(\mathrm{~s}, 3 \mathrm{H}) ;{ }^{13} \mathrm{C} \mathrm{NMR}(100 \mathrm{MHz}$, $\left.\mathrm{CDCl}_{3}\right) \delta 141.2(\mathrm{~d}, J=1.6 \mathrm{~Hz}), 134.7,134.4,133.7,133.1,132.6(\mathrm{~d}, J=10.1 \mathrm{~Hz}), 131.9(\mathrm{~d}, J=9.2 \mathrm{~Hz})$, $131.9(\mathrm{~d}, J=2.7 \mathrm{~Hz}), 131.9(\mathrm{~d}, J=2.7 \mathrm{~Hz}), 131.2,130.2,128.6,128.5,128.3,128.2,128.1,127.7,46.8$ $(\mathrm{d}, J=4.6 \mathrm{~Hz}),-4.6,-5.1 ;{ }^{31} \mathrm{P}$ NMR $\left(162 \mathrm{MHz}, \mathrm{CDCl}_{3}\right) \delta 25.0$; LRMS (MALDI-TOF): Mass calculated for $\mathrm{C}_{27} \mathrm{H}_{27} \mathrm{ClNOPSi}[\mathrm{M}]+:$ 476.0. Found 476.5.

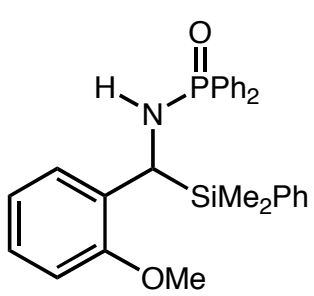

$\boldsymbol{P}, \boldsymbol{P}$-diphenyl- $\boldsymbol{N}$-(1-dimethylphenylsilyl-2-methoxyphenylmethylene)-phosphinic amide (13): Purified with $80 \%$ ethyl acetate/hexanes to yield $144 \mathrm{mg}$ (90\% yield) of colorless crystals. Mp: $101-102{ }^{\circ} \mathrm{C} ; \mathrm{R}_{f}=0.47$ (80/20 ethyl acetate/hexanes); IR (film) 3378, 3205, 1496, 1434, 1233, 1186, $1110 \mathrm{~cm}^{-1} ;{ }^{1} \mathrm{H} \mathrm{NMR}\left(400 \mathrm{MHz}, \mathrm{CDCl}_{3}\right)$ 7.70-7.53 (m, 4H), 7.46-7.16 (m, 11H), 7.12-7.03 (m, 1H), 6.88-6.76 (m, 2H), 6.63 $($ ap d, $J=7.9 \mathrm{~Hz}, 1 \mathrm{H}), 4.27(\mathrm{~m}, 1 \mathrm{H}), 3.43(\mathrm{~m}, 4 \mathrm{H}), 0.405(\mathrm{~s}, 3 \mathrm{H}), 0.214(\mathrm{~s}, 3 \mathrm{H}) ;{ }^{13} \mathrm{C}$ NMR $\left(100 \mathrm{MHz}, \mathrm{CDCl}_{3}\right) \delta 155.8,136.2,134.6,133.8,133.0,132.6(\mathrm{~d}, J=9.2 \mathrm{~Hz})$, $131.9(\mathrm{~d}, J=9.2 \mathrm{~Hz}), 131.7,131.6(\mathrm{~d}, J=1.8 \mathrm{~Hz}), 131.4(\mathrm{~d}, J=2.7 \mathrm{~Hz}), 131.2,129.5,128.3(\mathrm{~d}, J=12.8$ $\mathrm{Hz}), 128.0(\mathrm{~d}, J=12.8 \mathrm{~Hz}), 127.8,126.6,120.4,109.9,77.4,54.7,-4.1,-4.9 ;{ }^{31} \mathrm{P} \mathrm{NMR}(162 \mathrm{MHz}$, $\mathrm{CDCl}_{3}$ ) $\delta$ 25.9; LRMS (MALDI-TOF): Mass calculated for $\mathrm{C}_{28} \mathrm{H}_{30} \mathrm{NO}_{2} \mathrm{PSi}[\mathrm{M}+\mathrm{H}]+$ : 472.6. Found 472.9.

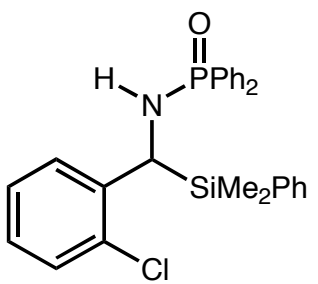

$\boldsymbol{P}, \boldsymbol{P}$-diphenyl- $\boldsymbol{N}$-(1-dimethylphenylsilyl-2-chlorophenylmethylene)-phosphinic amide (16): Purified with $80 \%$ ethyl acetate/hexanes to yield $76 \mathrm{mg}$ (55\% yield) of colorless crystals. Mp: 114-116; $\mathrm{R}_{f}=0.57$ (80/20 ethyl acetate/hexanes); IR (film) 3366, 3205, 3050, $1436 \mathrm{~cm}^{-1}$; ${ }^{1} \mathrm{H}$ NMR $\left(400 \mathrm{MHz}, \mathrm{CDCl}_{3}\right) \delta$ 7.65-6.90 (m, 19H), $4.48(\mathrm{dd}, J=10.4,10.4 \mathrm{~Hz}, 1 \mathrm{H}), 3.21(\mathrm{~m}, 1 \mathrm{H}), 0.490(\mathrm{~s}, 3 \mathrm{H}), 0.211(\mathrm{~s}, 3 \mathrm{H}) ;{ }^{13} \mathrm{C}$ NMR $\left(100 \mathrm{MHz}, \mathrm{CDCl}_{3}\right) \delta 140.7,134.8,134.4,132.7,132.4(\mathrm{~d}, J=9.2 \mathrm{~Hz}), 131.9$ $(\mathrm{d}, J=9.2 \mathrm{~Hz}), 131.8,131.8,131.7,131.5,131.1,130.1,129.0,128.4(\mathrm{~d}, J=11.9$ $\mathrm{Hz}), 128.3,128.0(\mathrm{~d}, J=3.0 \mathrm{~Hz}), 126.7,126.6,42.9(\mathrm{~d}, J=3.0 \mathrm{~Hz}),-4.1,-5.6(\mathrm{~d}, J=2.0 \mathrm{~Hz}) ;{ }^{31} \mathrm{P}$ NMR $\left(162 \mathrm{MHz}, \mathrm{CDCl}_{3}\right) \delta 25.3$; LRMS (MALDI-TOF): Mass calculated for $\mathrm{C}_{27} \mathrm{H}_{27} \mathrm{ClNOPSi}[\mathrm{M}+\mathrm{H}]+$ : 477.0. Found 477.6. 


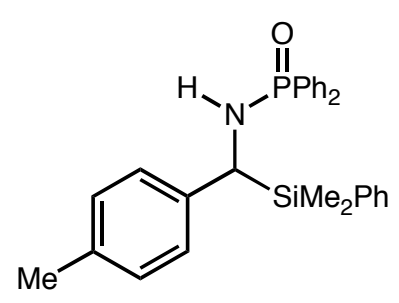

$P, P$-diphenyl- $N$-(1-dimethylphenylsilyl-4-methylphenylmethylene)phosphinic amide (12): Purified with $80 \%$ ethyl acetate/hexanes to yield 112 mg ( $75 \%$ yield) of colorless crystals. Mp: $147-149^{\circ} \mathrm{C} ; \mathrm{R}_{f}=0.50$ (80/20 ethyl acetate/hexanes); IR (film) 3203, 3049, 1432, 1255, 1182, 1108, 832, 747, $\mathrm{cm}^{-1}$; ${ }^{1} \mathrm{H}$ NMR (400 MHz, $\mathrm{CDCl}_{3}$ ) $\delta$ 7.68-7.58 (m, 4H), 7.46-7.27 (m, 9H), 7.26-7.18 (m, 2), 6.96 (ap d, $J=7.3 \mathrm{~Hz}, 2 \mathrm{H}), 6.70$ (ap d, $J=7.3 \mathrm{~Hz}, 2 \mathrm{H}), 3.90$ (dd, $J=$ 11.0, $11.0 \mathrm{~Hz}, 1 \mathrm{H}), 3.21(\mathrm{dd}, J=9.2,9.8 \mathrm{~Hz}, 1 \mathrm{H}), 2.28(\mathrm{~s}, 3 \mathrm{H}), 0.431(\mathrm{~s}, 3 \mathrm{H})$, $0.216(\mathrm{~s}, 3 \mathrm{H}) ;{ }^{13} \mathrm{C}$ NMR $\left(100 \mathrm{MHz}, \mathrm{CDCl}_{3}\right) \delta 144.4(\mathrm{~d}, J=1.8 \mathrm{~Hz}), 140.0(\mathrm{~d}, J=9.1 \mathrm{~Hz}), 139.8,138.5$, 137.9, $137.7(\mathrm{~d}, J=10.0 \mathrm{~Hz}), 137.3,137.0(\mathrm{~d}, J=9.1 \mathrm{~Hz}), 136.7(\mathrm{~d}, J=2.8 \mathrm{~Hz}), 136.6(\mathrm{~d}, J=2.7 \mathrm{~Hz})$, $134.9,133.7,133.4(\mathrm{~d}, J=12.8 \mathrm{~Hz}), 133.2(\mathrm{~d}, J=12.8 \mathrm{~Hz}), 133.0,131.3,46.9(\mathrm{~d}, J=4.5 \mathrm{~Hz}), 21.2$, $0.582,-0.009(\mathrm{~d}, J=1.8 \mathrm{~Hz}) ;{ }^{31} \mathrm{P}$ NMR $\left(162 \mathrm{MHz}, \mathrm{CDCl}_{3}\right) \delta 25.3$; LRMS (MALDI-TOF): Mass calculated for $\mathrm{C}_{28} \mathrm{H}_{30} \mathrm{NOPSi}[\mathrm{M}+\mathrm{H}]+:$ 456.6. Found 457.1.

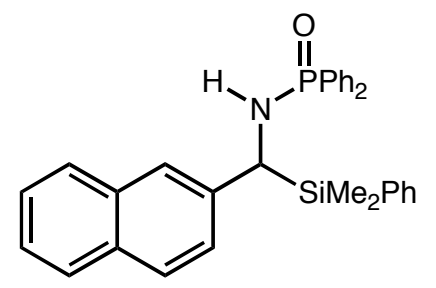

$P, P$-diphenyl- $N$-(1-dimethylphenylsilyl-2-naphthylmethylene)-phosphinic amide (10): Purified with $80 \%$ ethyl acetate/hexanes to yield $77 \mathrm{mg}(61 \%$ yield) of colorless crystals. Mp: $158-161{ }^{\circ} \mathrm{C} ; \mathrm{R}_{f}=0.50$ (80/20 ethyl acetate/hexanes); IR (film) 3418, 3067, 1649, 1631, 1154, $1105 \mathrm{~cm}^{-1} ;{ }^{1} \mathrm{H}$ NMR (400 MHz, $\left.\mathrm{CDCl}_{3}\right)$ ठ 7.79-7.74 (m, 1H), 7.68-7.57 (m, 6H), 7.44-7.26 (m, 11 $\mathrm{H}), 7.22-7.11(\mathrm{~m}, 3 \mathrm{H}), 7.00-6.94(\mathrm{~m}, 1 \mathrm{H}), 4.12(\mathrm{dd}, J=11.0,11.0 \mathrm{~Hz}, 1 \mathrm{H})$, $3.33(\mathrm{dd}, J=9.2,9.2 \mathrm{~Hz}, 1 \mathrm{H}), 0.475(\mathrm{~s}, 3 \mathrm{H}), 0.227(\mathrm{~s}, 3 \mathrm{H}) ;{ }^{13} \mathrm{C} \mathrm{NMR}\left(100 \mathrm{MHz}, \mathrm{CDCl}_{3}\right) \delta 140.2(\mathrm{~d}, J=$ $1.5 \mathrm{~Hz}), 134.8,134.7,133.4,133.3,132.6(\mathrm{~d}, J=9.2 \mathrm{~Hz}), 132.2,132.0,131.9(\mathrm{~d}, J=9.2 \mathrm{~Hz}), 131.8(\mathrm{~d}, J$ $=1.8 \mathrm{~Hz}), 131.7(\mathrm{~d}, J=2.7 \mathrm{~Hz}), 131.4,130.0,128.5(\mathrm{~d}, J=11.9 \mathrm{~Hz}), 128.2,128.1,127.6,127.5,125.9$, $125.4,125.1,124.5,47.4(\mathrm{~d}, J=3.0 \mathrm{~Hz}),-4.42,-4.94(\mathrm{~d}, J=3.0 \mathrm{~Hz}) ;{ }^{31} \mathrm{P}$ NMR $\left(162 \mathrm{MHz}, \mathrm{CDCl}_{3}\right) \delta$ 25.2; LRMS (MALDI-TOF): Mass (M+1) calculated for $\mathrm{C}_{31} \mathrm{H}_{30} \mathrm{NOPSi}[\mathrm{M}+\mathrm{H}]+:$ 492.6. Found 493.1.

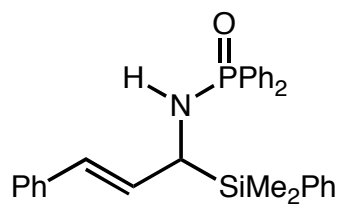

$\boldsymbol{P}, \boldsymbol{P}$-diphenyl- $\boldsymbol{N}$-(1-dimethylphenylsilyl-3-phenyl-2-propene)-phosphinic amide (19): Purified with $80 \%$ ethyl acetate/hexanes to yield $93 \mathrm{mg}$ (58\% yield) of colorless crystals. Mp: $60-62{ }^{\circ} \mathrm{C} ; \mathrm{R}_{f}=0.60$ (80/20 ethyl acetate/hexanes); IR (film) $3365,3198,3055,2957,1437,1250,1189,1121,1111,963,908,814,727$ $\mathrm{cm}^{-1}$; ${ }^{1} \mathrm{H}$ NMR $\left(400 \mathrm{MHz}, \mathrm{CDCl}_{3}\right)$ 87.85-7.80 (m, 2H), 7.73-7.68 (m, 2H), 7.57$7.55(\mathrm{~m}, 2 \mathrm{H}), 7.48-7.17(\mathrm{~m}, 14 \mathrm{H}), 6.24-6.10(\mathrm{~m}, 2 \mathrm{H}), 3.65(\mathrm{dt}, J=6.7,9.8 \mathrm{~Hz}, 1 \mathrm{H}), 2.85(\mathrm{dd}, J=8.9,8.5$ $\mathrm{Hz}, 1 \mathrm{H}), 0.48(\mathrm{~s}, 3 \mathrm{H}), 0.42(\mathrm{~s}, 3 \mathrm{H}) ;{ }^{13} \mathrm{C}$ NMR $\left(100 \mathrm{MHz}, \mathrm{CDCl}_{3}\right) \delta 137.6,135.1,134.5,133.3$ (d, $J=11.9$ $\mathrm{Hz}), 132.5(\mathrm{~d}, J=9.2 \mathrm{~Hz}), 132.0(\mathrm{~d}, J=9.2 \mathrm{~Hz}), 131.8,131.8,131.8,130.9(\mathrm{~d}, J=3.7 \mathrm{~Hz}), 130.0,128.6$, $128.5(\mathrm{~d}, J=3.7 \mathrm{~Hz}), 128.3,128.2,127.6,126.9,126.2,27.7(\mathrm{~d}, J=4.5 \mathrm{~Hz}),-4.86(\mathrm{~d}, J=2.7 \mathrm{~Hz}),-4.97$ $(\mathrm{d}, J=2.7 \mathrm{~Hz}) ;{ }^{31} \mathrm{P}$ NMR $\left(162 \mathrm{MHz}, \mathrm{CDCl}_{3}\right) \delta 24.6 ;{ }^{29} \mathrm{Si} \mathrm{NMR}\left(99 \mathrm{MHz}, \mathrm{CDCl}_{3}\right) \delta-0.997(\mathrm{~d}, J=12.2$ $\mathrm{Hz}$ ); LRMS (MALDI-TOF): Mass calculated for $\mathrm{C}_{29} \mathrm{H}_{30} \mathrm{NOPSi}[\mathrm{M}]+:$ 467.6. Found 467.6.

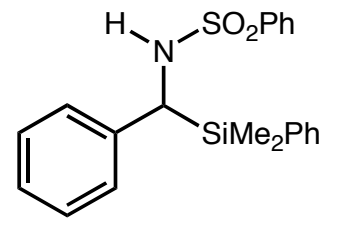

Phenylsulfonyl- $N$-(1-dimethylphenylsilyl-phenylmethylene)-phosphinic amide (6): Purified with $20 \%$ ethyl acetate/hexanes to yield $106 \mathrm{mg}$ (63\% yield) of colorless crystals. Mp: $157-159{ }^{\circ} \mathrm{C} ; \mathrm{R}_{f}=0.50$ (25/75 ethyl acetate/hexanes); IR (film) 3287, 3062, 2994, 1454, 1422, 1318, $1160 \mathrm{~cm}^{-1} ;{ }^{1} \mathrm{H}$ NMR (400 MHz, $\left.\mathrm{CDCl}_{3}\right)$ $\delta$ 7.52-7.13 (m, 10H), 7.04-6.95 (m, 3H), 6.72-6.66 (m, 2H), $4.89(\mathrm{~d}, J=7.9 \mathrm{~Hz}$, $1 \mathrm{H}), 4.16(\mathrm{~d}, J=8.5 \mathrm{~Hz}, 1 \mathrm{H}), 0.292(\mathrm{~s}, 3 \mathrm{H}), 0.207(\mathrm{~s}, 3 \mathrm{H}) ;{ }^{13} \mathrm{C} \mathrm{NMR}\left(100 \mathrm{MHz}, \mathrm{CDCl}_{3}\right) \delta$ 140.4, 138.7, $134.5,133.8,132.2,130.2,128.6,128.3,128.0,127.3,126.4,125.9,50.0,-4.6,-5.4 ;{ }^{29} \mathrm{Si}$ NMR $(99 \mathrm{MHz}$, 
$\left.\mathrm{CDCl}_{3}\right) \delta-0.961$; LRMS (MALDI-TOF): Mass calculated for $\mathrm{C}_{21} \mathrm{H}_{23} \mathrm{NO}_{2} \mathrm{SSi}[\mathrm{M}+\mathrm{Na}]+$ : 404.6. Found 404.9.<smiles>CCOC(=O)NC(c1ccccc1)c1ccccc1</smiles>

$\boldsymbol{P}, \boldsymbol{P}$-diethoxy- $N$-(1-dimethylphenylsilyl-phenylmethylene)-phosphinic amide (8): Purified with $40 \%$ ethyl acetate/hexanes to yield $232 \mathrm{mg}$ (53\% yield) of colorless crystals. Mp: $72.5-73.5^{\circ} \mathrm{C} ; \mathrm{R}_{f}=0.70$ (50/50 ethyl acetate/hexanes); IR (film) 3209 , 2979, 1228, 1053, 1024, 964, $693 \mathrm{~cm}^{-1} ;{ }^{1} \mathrm{H}$ NMR (400 MHz, $\left.\mathrm{CDCl}_{3}\right) \delta$ 7.40-7.28 (m, $5 \mathrm{H}), 7.22-7.02(\mathrm{~m}, 3 \mathrm{H}), 6.97-6.90(\mathrm{~m}, 2 \mathrm{H}), 3.92-3.77(\mathrm{~m}, 2 \mathrm{H}), 3.75-3.66(\mathrm{~m}, 2 \mathrm{H})$, $3.42-3.22(\mathrm{~m}, 2 \mathrm{H}), 1.18(\mathrm{t}, J=7.5 \mathrm{~Hz}, 3 \mathrm{H}), 0.779(\mathrm{t}, J=6.7 \mathrm{~Hz}, 3 \mathrm{H}), 0.301(\mathrm{~s}, 3 \mathrm{H})$, 0.217 (s, 3H); ${ }^{13} \mathrm{C}$ NMR $\left(100 \mathrm{MHz}, \mathrm{CDCl}_{3}\right) \delta 142.6,134.51,134.47,129.8,127.89,127.85,126.3,125.6$, $62.1,62.02,61.98,47.6(\mathrm{~d}, J=5.5 \mathrm{~Hz}), 16.3(\mathrm{~d}, J=7.3), 15.6(\mathrm{~d}, J=8.2 \mathrm{~Hz}),-4.7,-5.4(\mathrm{~d}, J=3.7) ;{ }^{31} \mathrm{P}$ NMR (162 MHz, $\left.\mathrm{CDCl}_{3}\right) \delta 9.29 ;{ }^{29} \mathrm{Si} \mathrm{NMR}\left(99 \mathrm{MHz}, \mathrm{CDCl}_{3}\right) \delta-1.2(\mathrm{~d}, J=14.6 \mathrm{~Hz})$; LRMS (MALDI-TOF): Mass (M+Na+H) calculated for $\mathrm{C}_{19} \mathrm{H}_{28} \mathrm{NO}_{3} \mathrm{PSi}[\mathrm{M}+\mathrm{Na}+\mathrm{H}]=:$ 401.5. Found 401.3.

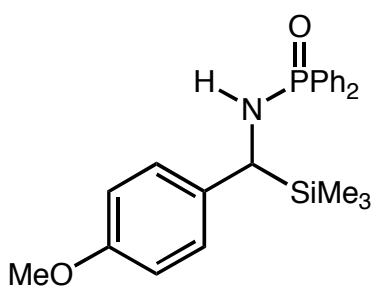

$\boldsymbol{P}, \boldsymbol{P}$-diphenyl- $\boldsymbol{N}$-(1-trimethylsilyl-4-methoxyphenylmethylene)-phosphinic amide (20): Purified with 50\% ethyl acetate/hexanes to yield $144 \mathrm{mg}(67 \%$ yield) of colorless crystals. Mp: $133-134{ }^{\circ} \mathrm{C} ; \mathrm{R}_{f}=0.43(50 / 50$ ethyl acetate/hexanes); IR (film) 3209, 2957, 1515, 1431, 1241, 1171, $1039 \mathrm{~cm}^{-1} ;{ }^{1} \mathrm{H}$ NMR $\left(400 \mathrm{MHz}, \mathrm{CDCl}_{3}\right) \delta$ 7.83-7.61 (m, 4H), 7.47-7.16 (m, 6H), 6.85-6.68 (m, $4 \mathrm{H}), 3.75-3.66(\mathrm{~m}, 4 \mathrm{H}), 3.25(\mathrm{dd}, J=8.5,8.5 \mathrm{~Hz}, 1 \mathrm{H}), 0.00(\mathrm{~s}, 9 \mathrm{H}) ;{ }^{13} \mathrm{C} \mathrm{NMR}$ $\left(100 \mathrm{MHz}, \mathrm{CDCl}_{3}\right) \delta 157.5,135.2(\mathrm{~d}, J=2.5 \mathrm{~Hz}), 133.8,132.8(\mathrm{~d}, J=10.1 \mathrm{~Hz})$, 132.6, $132.0(\mathrm{~d}, J=9.2 \mathrm{~Hz}), 131.8(\mathrm{~d}, J=1.8 \mathrm{~Hz}), 131.6(\mathrm{~d}, J=1.8 \mathrm{~Hz}), 131.3,128.5(\mathrm{~d}, J=11.9 \mathrm{~Hz})$, $128.1(\mathrm{~d}, J=12.8 \mathrm{~Hz}), 127.2,55.4,46.7(\mathrm{~d}, \mathrm{~J}=3.6 \mathrm{~Hz}),-3.4(\mathrm{~d}, J=6.4 \mathrm{~Hz}) ;{ }^{31} \mathrm{P}$ NMR $\left(162 \mathrm{MHz}, \mathrm{CDCl}_{3}\right)$ $\delta$ 25.0; ${ }^{29} \mathrm{Si} \mathrm{NMR}\left(99 \mathrm{MHz}, \mathrm{CDCl}_{3}\right) \delta 5.02$ (d, $J=9.2 \mathrm{~Hz}$ ); LRMS (MALDI-TOF): Mass calculated for $\mathrm{C}_{23} \mathrm{H}_{28} \mathrm{NO}_{2} \mathrm{PSi}[\mathrm{M}+\mathrm{H}]+:$ 410.5. Found 410.8.

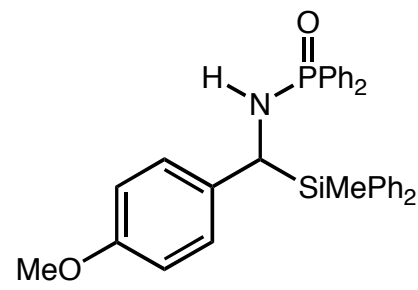

$\boldsymbol{P}, \boldsymbol{P}$-diphenyl- $N$-(1-methyldiphenylsilyl-4-methoxyphenylmethylene)phosphinic amide (21): Purified with 80\% ethyl acetate/hexanes to yield 137 $\mathrm{mg}(80 \%$ yield $)$ of colorless crystals. Mp: $165-166^{\circ} \mathrm{C} ; \mathrm{R}_{f}=0.32(80 / 20$ ethyl acetate/hexanes); IR (film) 3372, 3220, 3060, 1502, 1447, 1257, 1168, 1109 $\mathrm{cm}^{-1} ;{ }^{1} \mathrm{H}$ NMR $\left(400 \mathrm{MHz}, \mathrm{CDCl}_{3}\right) \delta$ 7.61-7.18 (m, 20H), 6.70-6.61 (m, 4H), $4.31(\mathrm{dd}, J=10.4,10.4, \mathrm{~Hz}, 1 \mathrm{H}), 3.73(\mathrm{~s}, 3 \mathrm{H}), 3.29(\mathrm{dd}, J=9.5,9.5 \mathrm{~Hz}, 1 \mathrm{H})$, 0.465 (s, 3H); ${ }^{13} \mathrm{C} \mathrm{NMR}\left(100 \mathrm{MHz}, \mathrm{CDCl}_{3}\right) \delta 157.7,135.7,135.5,134.2,133.8$, $133.3,133.3,132.9,132.6(\mathrm{~d}, J=9.2 \mathrm{~Hz}), 132.1,131.9(\mathrm{~d}, J=10.1 \mathrm{~Hz}), 131.7(\mathrm{~d}, J=2.7 \mathrm{~Hz}), 131.6(\mathrm{~d}, J$ $=2.7 \mathrm{~Hz}), 130.0(\mathrm{~d}, J=13.7 \mathrm{~Hz}), 128.4(\mathrm{~d}, J=12.8 \mathrm{~Hz}), 128.1,128.1,128.0,128.0,113.4,55.4,45.0(\mathrm{~d}$, $J=4.6 \mathrm{~Hz}),-5.08 ;{ }^{31} \mathrm{P}$ NMR $\left(162 \mathrm{MHz}, \mathrm{CDCl}_{3}\right) \delta 25.6 ;{ }^{29} \mathrm{Si} \mathrm{NMR}\left(99 \mathrm{MHz}, \mathrm{CDCl}_{3}\right) \delta-7.23(\mathrm{~d}, J=13.0$ $\mathrm{Hz}$ ); LRMS (MALDI-TOF): Mass (M+1) calculated for $\mathrm{C}_{33} \mathrm{H}_{32} \mathrm{NO}_{2} \mathrm{PSi}[\mathrm{M}+\mathrm{H}]+:$ 534.7. Found 535.2.

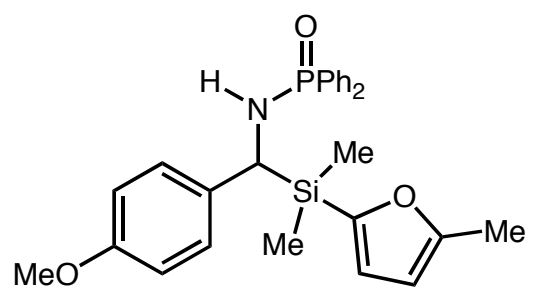

$P, P$-diphenyl- $N$-(1-dimethyl--(4-methyl)2-furylsilyl-4methoxyphenylmethylene)-phosphinic amide (24): Purified with $80 \%$ ethyl acetate/hexanes to yield $48 \mathrm{mg}$ (32\% yield) of colorless crystals. Mp: $129.5-131{ }^{\circ} \mathrm{C} ; \mathrm{R}_{f}=0.35$ (80/20 ethyl acetate/hexanes); IR (film) 3376, 3198, 1504, 1432, 1275, 1243, $745 \mathrm{~cm}^{-1} ;{ }^{1} \mathrm{H}$ NMR (400 $\left.\mathrm{MHz}, \mathrm{CDCl}_{3}\right) \delta$ 7.72-7.63 (m, 4H), 7.45-7.20 (m, 6H), 6.75-6.62 (m, 4H), 6.52 (ap d, $J=3.0 \mathrm{~Hz}, 1 \mathrm{H}), 5.99(\mathrm{~s}, 1 \mathrm{H}), 3.87-3.66(\mathrm{~m}, 5 \mathrm{H}), 2.27$ 
(s, 3H), $0.381(\mathrm{~s}, 3 \mathrm{H}), 0.062(\mathrm{~s}, 3 \mathrm{H}) ;{ }^{13} \mathrm{C} \mathrm{NMR}\left(100 \mathrm{MHz}, \mathrm{CDCl}_{3}\right) \delta 157.7(\mathrm{~d}, J=5.5 \mathrm{~Hz}), 154.3,134.6$, $133.7,133.2,132.7$ (d, $J=10.1 \mathrm{~Hz}), 132.0(\mathrm{~d}, J=9.2 \mathrm{~Hz}), 131.9,131.8(\mathrm{~d}, J=2.7 \mathrm{~Hz}), 131.7(\mathrm{~d}, J=1.8$ $\mathrm{Hz}), 128.5(\mathrm{~d}, J=12.8 \mathrm{~Hz}), 128.2(\mathrm{~d}, J=11.9 \mathrm{~Hz}), 127.6,124.0,113.5,106.4,55.4,46.3(\mathrm{~d}, J=3.7 \mathrm{~Hz})$, 14.0, -4.10, -5.40; ${ }^{31} \mathrm{P}$ NMR (162 $\left.\mathrm{MHz}, \mathrm{CDCl}_{3}\right) \delta 25.2 ;{ }^{29} \mathrm{Si}$ NMR $\left(99 \mathrm{MHz}, \mathrm{CDCl}_{3}\right) \delta-8.58(\mathrm{~d}, J=11.6$ $\mathrm{Hz}$ ); LRMS (MALDI-TOF): Mass calculated for $\mathrm{C}_{27} \mathrm{H}_{30} \mathrm{NO}_{3} \mathrm{PSi}$ [M+Na]: 498.6. Found 499.0.

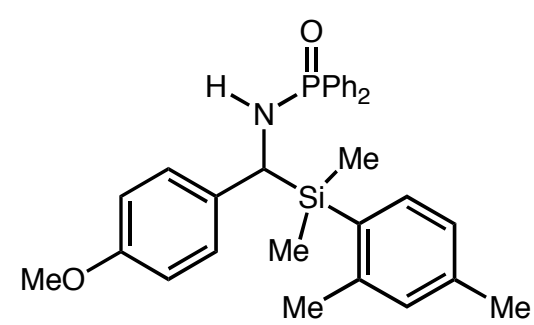

$P, P$-diphenyl- $N$-(1-dimethyl--(2,4-dimethyl)-phenylsilyl-4methoxyphenylmethylene)-phosphinic amide (23): Purified with $50 \%$ ethyl acetate/hexanes to yield $50 \mathrm{mg}$ (53\% yield) of colorless crystals. Mp: $137-139{ }^{\circ} \mathrm{C} ; \mathrm{R}_{f}=0.43$ (50/50 ethyl acetate/hexanes); IR (film) 3367, 3215, 3055, 2955, 1605, 1508, 1438, 1246, 1192, 1117, $1065,1035,838,806,727,700 \mathrm{~cm}^{-1} ;{ }^{1} \mathrm{H}$ NMR (400 MHz, $\left.\mathrm{CDCl}_{3}\right) \delta$ 7.62-7.49 (m, $4 \mathrm{H}), 7.46-7.16(\mathrm{~m}, 7 \mathrm{H}), 7.04-6.97(\mathrm{~m}, 2 \mathrm{H}), 6.84-6.68(\mathrm{~m}$, 4H), $9.98(\mathrm{dd}, J=10.8,10.8 \mathrm{~Hz}, 1 \mathrm{H}), 3.76(\mathrm{~s}, 3 \mathrm{H}), 3.19(\mathrm{dd}, J=9.6,9.6 \mathrm{~Hz}, 1 \mathrm{H}), 2.34(\mathrm{~s}, 3 \mathrm{H}), 2.17(\mathrm{~s}$, $3 \mathrm{H}), 0.347$ (s, 3H), $0.312(\mathrm{~s}, 3 \mathrm{H}) ;{ }^{13} \mathrm{C}$ NMR $\left(100 \mathrm{MHz}, \mathrm{CDCl}_{3}\right) \delta 157.6,144.5,140.0,135.9,134.7(\mathrm{~d}, J=$ $1.8 \mathrm{~Hz}), 133.2,133.1,132.5(\mathrm{~d}, J=9.2 \mathrm{~Hz}), 132.0(\mathrm{~d}, J=10.1 \mathrm{~Hz}), 131.9(\mathrm{~d}, J=12.8 \mathrm{~Hz}), 131.6(\mathrm{~d}, J=$ $2.7 \mathrm{~Hz}), 131.4,130.0,128.4(\mathrm{~d}, J=12.8 \mathrm{~Hz}), 128.1(\mathrm{~d}, J=12.8 \mathrm{~Hz}), 127.2,126.0 .113 .5,55.4,46.4(\mathrm{~d}, J$ $=4.6 \mathrm{~Hz}), 23.6,21.5,-2.4,-3.3 ;{ }^{31} \mathrm{P} \mathrm{NMR}\left(162 \mathrm{MHz}, \mathrm{CDCl}_{3}\right) \delta 24.92 ;{ }^{29} \mathrm{Si} \mathrm{NMR}\left(99 \mathrm{MHz}, \mathrm{CDCl}_{3}\right) \delta$ $-0.017\left(\mathrm{~d}, J=12.2 \mathrm{~Hz}\right.$ ); LRMS (MALDI-TOF): Mass calculated for $\mathrm{C}_{30} \mathrm{H}_{34} \mathrm{NO}_{2} \mathrm{PSi}[\mathrm{M}]+:$ 499.7. Found 499.8 .

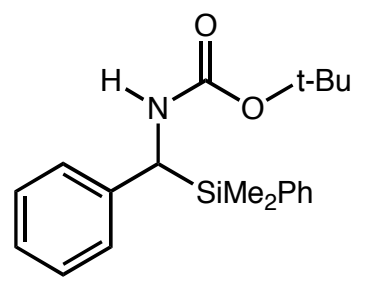

tert-Butyl dimethylphenylsilyl(phenylmethylene)carbamate (32): Purified with $10 \%$ ethyl acetate/hexanes to yield $56 \mathrm{mg}$ (82\% yield) of colorless crystals. Mp: $72-73{ }^{\circ} \mathrm{C} ; \mathrm{R}_{f}=0.82$ (25/75 ethyl acetate/hexanes); IR (film) $3745,3276,2972$, 1696, 1489, 1489, 1365, 1250, 1169, 1113, 1003, 834, 735, $700 \mathrm{~cm}^{-1} ;{ }^{1} \mathrm{H}$ NMR $\left(400 \mathrm{MHz}, \mathrm{CDCl}_{3},\right) \delta$ (major rotomer) 7.44-7.32 (m, 5H), 7.26-7.20 (m, 2H), 7.17$7.12(\mathrm{~m}, 1 \mathrm{H}), 6.98-6.92(\mathrm{~m}, 2 \mathrm{H}), 4.91(\mathrm{~m}, 1 \mathrm{H}), 4.52(\mathrm{~d}, J=7.3 \mathrm{~Hz}, 1 \mathrm{H}), 1.40(\mathrm{~s}$, 9H), 0.343 (s, 3H), $0.271(\mathrm{~s}, 3 \mathrm{H}) ;{ }^{13} \mathrm{C} \mathrm{NMR}\left(100 \mathrm{MHz}, \mathrm{CDCl}_{3}\right) \delta$ 156.1, 141.7, $135.1,134.5,129.9,128.2,128.1,126.0,125.7,79.7,47.1,28.6,-4.39,-4.70 ;{ }^{29} \mathrm{Si} \mathrm{NMR}(99 \mathrm{MHz}$, $\left.\mathrm{CDCl}_{3}\right) \delta-1.89$; LRMS (MALDI-TOF): Mass calculated for $\mathrm{C}_{20} \mathrm{H}_{27} \mathrm{NO}_{2} \mathrm{Si}$ [M+Na]+: 364.5. Found 364.6.

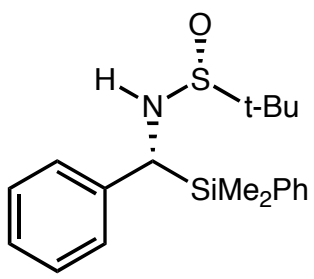

$\left.\mathbf{R}_{\mathrm{s}}, \mathbf{R}\right)-\boldsymbol{N}$-(1-dimethylphenylsilylphenylmethylene)-t-butanesulfinamide (25): Purified with $40 \%$ ethyl acetate/hexanes to yield $124 \mathrm{mg}$ (73\% yield) of colorless crystals Mp: $91-92{ }^{\circ} \mathrm{C} ;[\alpha] \mathrm{D}=53.2\left(c \quad 0.47, \mathrm{CH}_{2} \mathrm{Cl}_{2}\right) ; \mathrm{R}_{f}=0.57$ (50/50 ethyl acetate/hexanes); IR (film) 3430, 3245, 3063, 2956, 1250, 1114, 1062, 833, 814, 700 $\mathrm{cm}^{-1} ;{ }^{1} \mathrm{H} \mathrm{NMR}\left(400 \mathrm{MHz}, \mathrm{CDCl}_{3},\right) \delta 7.43-7.00(\mathrm{~m}, 10 \mathrm{H}), 4.08(\mathrm{~d}, J=8.5 \mathrm{~Hz}, 1 \mathrm{H})$, $3.49(\mathrm{~d}, J=8.5 \mathrm{~Hz}, 1 \mathrm{H}), 1.01(\mathrm{~s}, 9 \mathrm{H}), 0.375(\mathrm{~s}, 3 \mathrm{H}), 0.248(\mathrm{~s}, 3 \mathrm{H}) ;{ }^{13} \mathrm{C}$ NMR $(100$ $\left.\mathrm{MHz}_{,} \mathrm{CDCl}_{3}\right) \delta 141.2,134.7,134.4,129.9,128.1,127.9,126.7,126.1,56.6,52.9,22.5,-4.25,-5.06 ;{ }^{29} \mathrm{Si}$ NMR (99 MHz, $\mathrm{CDCl}_{3}$ ) $\delta-1.66$; LRMS (ESI) Mass calculated for $\mathrm{C}_{19} \mathrm{H}_{28} \mathrm{NO}_{2} \mathrm{SSi}$ [M]+: 346.6. Found 346.2; Optical rotation of enantiomer (ent-25, $\left(\mathbf{S}_{\mathbf{s}}, \mathbf{S}\right)$ : $[\alpha] \mathrm{D}=-50.0\left(c 0.47, \mathrm{CH}_{2} \mathrm{Cl}_{2}\right)$; Crystal data for ent$25\left(\mathbf{S}_{\mathrm{s}}, \mathbf{S}\right): \mathrm{C}_{19} \mathrm{H}_{27} \mathrm{NOSSi}, \mathrm{M}_{\mathrm{r}}=345.57$, crystal dimensions $=0.470 \times 0.068 \times 0.024 \mathrm{~mm}$, orthorhombic, space group P $2_{1} 2_{1} 2_{1}, a=6.4269(8), b=16.553(2), c=18.276(2) \AA a=6.4269(8), b=16.553(2), c=$ 18.276(2) $\AA, \alpha=\beta=\gamma=90.00^{\circ}, \mathrm{V}=1944(4) \AA^{3}, \mathrm{Z}=4, \rho_{\text {calcd }}=1.181 \mathrm{~g} \mathrm{~cm}^{-3}, 2 \theta_{\mu \alpha}=57.294$, number of measured reflections $=18115$, number of unique reflections $=4722$, number of parameters $=217, R(F)=$ $\Sigma\left\|F_{o}|-| F_{c}\right\| / \Sigma\left|F_{o}\right|$ for $F_{o}^{2}>2 \sigma\left(F_{o}{ }^{2}\right) . R_{w}\left(F^{2}\right)=\left\{\Sigma\left[\left(F_{o}{ }^{2}-F_{c}{ }^{2}\right)^{2}\right] / \Sigma w \mathrm{~F}_{o}{ }^{4}\right\}^{1 / 2}$ for all data, $w^{-1}=\sigma^{2}\left(F_{o}{ }^{2}\right)+$ $\left(0.0600 F_{o}{ }^{2}\right)^{2}$ for $F_{o}{ }^{2} \geq 0$ and $w^{-1}=\sigma^{2}\left(F_{o}{ }^{2}\right)$ for $F_{o}{ }^{2}<0, \mathrm{R}_{1}(F)=0.0620, \mathrm{wR}\left(F^{2}\right)=0.1353$, exposure time $=$ 20s/frame. The hydrogen atom positions were fixed geometrically at calculated distances and allowed to 
ride on the parent atoms, except the hydrogen on the nitrogen, which was located using difference electron density maps, the position was fixed and then was refined isotropically. Reference number for the Cambridge data base: CCDC 257698.<smiles>Cc1ccc([C@H](NC(=O)C(C)(C)C)c2ccccc2)cc1</smiles>

$\left(\mathbf{R}_{\mathrm{s}}, \mathbf{R}\right)-N$-(1-dimethylphenylsilyl-4-methylphenylmethylene)-tbutanesulfinamide (26): Purified with $40 \%$ ethyl acetate/hexanes to yield 112 $\mathrm{mg}\left(72 \%\right.$ yield) of colorless oil. $\mathrm{R}_{f}=0.60$ (50/50 ethyl acetate/hexanes); IR (film) $3315,3224,2955,1511,1250,1114,1064,824 \mathrm{~cm}^{-1} ;{ }^{1} \mathrm{H}$ NMR (400 $\mathrm{MHz}, \mathrm{CDCl}_{3}$,) $\delta$ 7.46-7.28 (m, 5H), 7.10-6.84 (m, 4H), $4.03(\mathrm{~d}, J=8.5 \mathrm{~Hz}, 1 \mathrm{H})$, $3.43(\mathrm{~d}, J=8.5 \mathrm{~Hz}, 1 \mathrm{H}), 2.30(\mathrm{~s}, 3 \mathrm{H}), 1.08(\mathrm{~s}, 9 \mathrm{H}), 0.365(\mathrm{~s}, 3 \mathrm{H}), 0.242(\mathrm{~s}, 3 \mathrm{H})$; ${ }^{13} \mathrm{C} \mathrm{NMR}\left(100 \mathrm{MHz} \mathrm{CDCl}_{3}\right) \delta 138.1,135.6,135.0,134.5,129.9,128.9,128.0$, 126.7, 56.6, 52.7, 22.5, 21.2, -4.10, $-5.04 ;{ }^{29} \mathrm{Si} \mathrm{NMR}\left(99 \mathrm{MHz}, \mathrm{CDCl}_{3}\right.$ ) $\delta$-2.02; LRMS (ESI) Mass calculated for $\mathrm{C}_{20} \mathrm{H}_{30} \mathrm{NOSSi}[\mathrm{M}]+:$ 360.6. Found 360.2.<smiles>COc1ccc([C@H](NC(=O)C(C)(C)C)c2ccccc2)cc1</smiles>

$\left(\mathbf{R}_{\mathrm{s}}, \mathbf{R}\right)-\mathrm{N}$-(1-dimethylphenylsilyl-4-methoxyphenylmethylene)-tbutanesulfinamide (27): Purified with $40 \%$ ethyl acetate/hexanes to yield $118 \mathrm{mg}(75 \%$ yield $)$ of colorless oil. $\mathrm{R}_{f}=0.57(50 / 50$ ethyl acetate/hexanes); IR (film) 3429, 3252, 2955, 1610, 1510, 1248, 1062, 1040, 834, $811 \mathrm{~cm}^{-1} ;{ }^{1} \mathrm{H}$ NMR (400 $\mathrm{MHz}, \mathrm{CDCl}_{3}$,) $\delta 7.42-7.28(\mathrm{~m}, 5 \mathrm{H})$, 6.98-6.92 (m, 2H), 6.82-6.74 (m, 2H), $4.00(\mathrm{~d}, J=8.5 \mathrm{~Hz}, 1 \mathrm{H}), 3.76(\mathrm{~s}$, $3 \mathrm{H}), 3.39(\mathrm{~d}, J=8.5 \mathrm{~Hz}, 1 \mathrm{H}), 1.08(\mathrm{~s}, 9 \mathrm{H}), 0.355(\mathrm{~s}, 3 \mathrm{H}), 0.233(\mathrm{~s}, 3 \mathrm{H}) ;{ }^{13} \mathrm{C}$ NMR $\left(100 \mathrm{MHz}, \mathrm{CDCl}_{3}\right) \delta 158.0,134.9,134.5,133.3,129.9,128.0,127.9,113.7,56.6,55.3,52.3,22.5$, -4.16, -5.00; ${ }^{29} \mathrm{Si}$ NMR $\left(99 \mathrm{MHz}, \mathrm{CDCl}_{3}\right) \delta$-2.04; LRMS (ESI) Mass calculated for $\mathrm{C}_{20} \mathrm{H}_{30} \mathrm{NO}_{2} \mathrm{SSi}[\mathrm{M}]+$ : 376.6. Found 376.2. 
<smiles>CC(C)(C)[S@](=O)N[C@@H](c1ccccc1)c1ccc2ccccc2c1</smiles>

$\left(\mathbf{R}_{\mathrm{s}}, \mathbf{R}\right)-\boldsymbol{N}$-(1-dimethylphenylsilyl-2-naphthylmethylene)- $t$ butanesulfinamide (29): Purified with $40 \%$ ethyl acetate/hexanes to yield 143 $\mathrm{mg}$ (90\% yield) of colorless oil. $\mathrm{R}_{f}=0.67$ (50/50 ethyl acetate/hexanes); IR (film) $3327,3227,3051,2957,1250,1114,1067,853,820,737 \mathrm{~cm}^{-1}$; ${ }^{1} \mathrm{H} \mathrm{NMR}$ $\left(400 \mathrm{MHz}, \mathrm{CDCl}_{3}\right.$ ) $\delta$ 7.82-7.70 (m, 3H), 7.54-7.30 (m, 8H), 7.21-7.14 (m, $1 \mathrm{H}), 4.26(\mathrm{~d}, J=8.5 \mathrm{~Hz}, 1 \mathrm{H}), 3.60(\mathrm{~d}, J=8.5 \mathrm{~Hz}, 1 \mathrm{H}), 1.12(\mathrm{~s}, 9 \mathrm{H}), 0.428(\mathrm{~s}$, $3 \mathrm{H}), 0.269$ (s, 3H); ${ }^{13} \mathrm{C} \mathrm{NMR}\left(100 \mathrm{MHz}, \mathrm{CDCl}_{3}\right) \delta 139.0,134.7,134.6,133.4,132.3,130.1,128.1,127.8$, $127.8,127.7,126.0,125.6,125.3,125.1,56.8,53.1,22.6,-4.09,-4.94 ;{ }^{29} \mathrm{Si} \mathrm{NMR}\left(99 \mathrm{MHz}, \mathrm{CDCl}_{3}\right) \delta$ -1.35; LRMS (ESI) Mass calculated for $\mathrm{C}_{23} \mathrm{H}_{30} \mathrm{NOSSi}[\mathrm{M}]+$ : 396.6. Found 396.2.

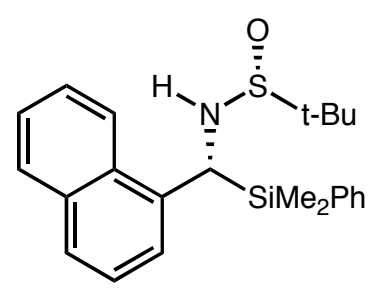

$\left(\mathbf{R}_{\mathrm{s}}, \mathbf{R}\right)-\mathrm{N}$-(1-dimethylphenylsilyl-1-naphthylmethylene)-t-butanesulfinamide (28): Purified with $20 \%$ ethyl acetate/hexanes to yield $76 \mathrm{mg}$ (60\% yield) of colorless oil. $\mathrm{R}_{f}=0.37$ (50/50 ethyl acetate/hexanes); IR (film) 3323, 3245, 3048, 2956, 1252, 1114, 1067, 833, 817, $776 \mathrm{~cm}^{-1} ;{ }^{1} \mathrm{H}$ NMR (400 MHz, $\mathrm{CDCl}_{3}$,) $\delta$ (major diastereomer) 8.00-7.92 (m, 1H), 7.88-7.82 (m, 1H), 7.73-7.66 (m, 1H), 7.50-7.22 (m, 9H), $5.07(\mathrm{~d}, J=7.3 \mathrm{~Hz}, 1 \mathrm{H}), 3.70(\mathrm{~d}, J=8.5 \mathrm{~Hz}, 1 \mathrm{H}), 1.13(\mathrm{~s}$, 9H), 0.408 (s, 3H), 0.135 (s, 3H); ${ }^{13} \mathrm{C} \mathrm{NMR}\left(100 \mathrm{MHz}, \mathrm{CDCl}_{3}\right) \delta$ 137.7, 134.5, 133.7, 130.7, 130.0, 129.0, 128.0, 126.6, 125.5, 125.5, 125.4, 125.4, 124.5, 123.0, 56.8, 53.2, 22.6, -3.57, $-4.66 ;{ }^{29} \mathrm{Si} \mathrm{NMR}\left(99 \mathrm{MHz}, \mathrm{CDCl}_{3}\right) \delta-0.445$; LRMS (ESI) Mass calculated for $\mathrm{C}_{23} \mathrm{H}_{30} \mathrm{NOSSi}[\mathrm{M}]+$ : 396.6. Found 396.2.

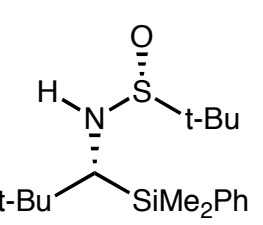

$\left(\mathbf{R}_{\mathrm{s}}, \mathbf{R}\right)-\boldsymbol{N}$-(1-dimethylphenylsilyl-2,2-dimethylpropyl)-t-butanesulfinamide (31): Purified with $40 \%$ ethyl acetate/hexanes to yield $120 \mathrm{mg}$ (72\% yield) of colorless crystals. Mp: $76-79{ }^{\circ} \mathrm{C} ;[\alpha]_{\mathrm{D}}{ }^{25}=13.4\left(c \quad 0.50, \mathrm{CH}_{2} \mathrm{Cl}_{2}\right) ; \mathrm{R}_{f}=0.67$ (50/50 ethyl acetate/hexanes); IR (film) 3429, 3376, 3121, 2955, 2867, 1248, 1015, $817 \mathrm{~cm}^{-1}$; ${ }^{1} \mathrm{H}$

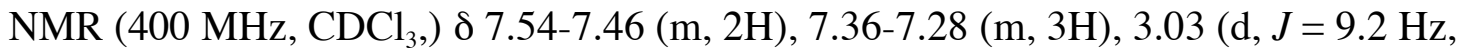
$1 \mathrm{H}), 2.90(\mathrm{~d}, J=9.2 \mathrm{~Hz}, 1 \mathrm{H}), 1.01(\mathrm{~s}, 9 \mathrm{H}), 0.966(\mathrm{~s}, 9 \mathrm{H}), 0.399(\mathrm{~s}, 3 \mathrm{H}), 0.385(\mathrm{~s}, 3 \mathrm{H}) ;{ }^{13} \mathrm{C}$ NMR $(100$ $\left.\mathrm{MHz}_{\mathrm{CDCl}}\right) \delta 138.8,134.2,129.3,128.1,59.2,56.8,35.8,29.6,23.0,-0.470,-1.28 ;{ }^{29} \mathrm{Si} \mathrm{NMR}$ (99 $\left.\mathrm{MHz}, \mathrm{CDCl}_{3}\right) \delta-4.11$; LRMS (ESI) Mass calculated for $\mathrm{C}_{17} \mathrm{H}_{32} \mathrm{NOSSi}[\mathrm{M}]+$ : 326.6. Found 326.2. Crystal data for 31: $\mathrm{C}_{17} \mathrm{H}_{31} \mathrm{NOSSi} \cdot \mathrm{H}_{2} \mathrm{O}: \mathrm{M}_{\mathrm{r}}=343.59$, crystal dimensions $=0.360 \times 0.098 \times 0.024 \mathrm{~mm}$, orthorhombic, space group P $2_{1} 2_{1} 2_{1}, a=6.5692(13), b=15.207(3), c=20.303(4) \AA, \alpha=\beta=\gamma=90.00^{\circ}$, $\mathrm{V}=2028.2(7) \AA^{3}, \mathrm{Z}=2, \rho_{\text {calcd }}=1.125 \mathrm{~g} \mathrm{~cm}^{-3}, 2 \theta_{\mu \alpha \xi}=43.952$, number of measured reflections $=18624$, number of unique reflections $=4936, \mu=0.225 \mathrm{~mm}^{-1}$, min $/$ max transmission $=0.93078 / 0.99184$, number of parameters $=201, R(F)=\Sigma|| F_{o}|-| F_{c} \| / \Sigma\left|F_{o}\right|$ for $F_{o}^{2}>2 \sigma\left(F_{o}^{2}\right) \cdot R_{w}\left(F^{2}\right)=\left\{\Sigma\left[\left(F_{o}^{2}-F_{c}^{2}\right)^{2}\right] / \Sigma w \mathrm{~F}_{\mathrm{o}}^{4}\right\}^{1 / 2}$ for all data, $w^{-1}=\sigma^{2}\left(F_{o}^{2}\right)+\left(0.0505 F_{o}^{2}\right)^{2}$ for $F_{o}^{2} \geq 0$ and $w^{-1}=\sigma^{2}\left(F_{o}{ }^{2}\right)$ for $F_{o}{ }^{2}<0, \mathrm{R}_{1}(F)=0.0592, \mathrm{wR}\left(F^{2}\right)=$ 0.1279 , exposure time $=35 \mathrm{~s} /$ frame. The hydrogen atom positions were fixed geometrically at calculated distances and allowed to ride on the parent atoms, except the hydrogen on the nitrogen, which was located using difference electron density maps, the position was fixed and then was refined isotropically. Reference number for the Cambridge data base: CCDC 257699.

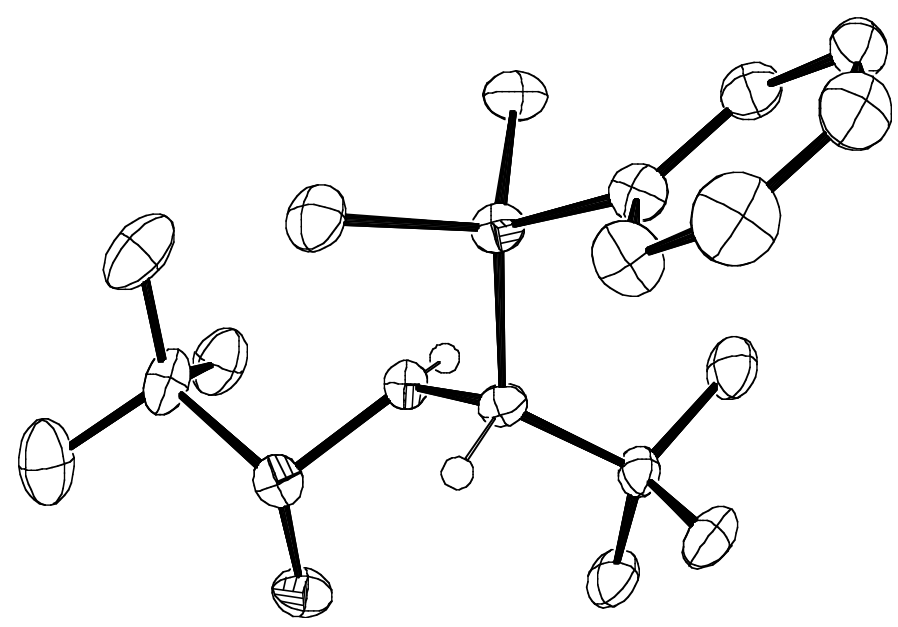




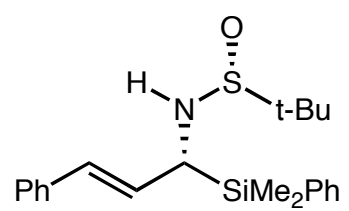

$9.2 \mathrm{~Hz}, 1 \mathrm{H}), 1.11(\mathrm{~s}, 9 \mathrm{H}), 0.439(\mathrm{~s}, 3 \mathrm{H}), 0.408(\mathrm{~s}, 3 \mathrm{H}) ;{ }^{13} \mathrm{C}$ NMR $\left(100 \mathrm{MHz}, \mathrm{CDCl}_{3}\right) \delta 137.4,135.0$, $134.3,130.4,130.1,128.7,128.5,128.2,127.1,126.4,56.5,51.9,22.6,-4.55,-4.65 ;{ }^{29} \mathrm{Si} \mathrm{NMR}(99 \mathrm{MHz}$, $\left.\mathrm{CDCl}_{3}\right) \delta-2.09$; LRMS (ESI) Mass calculated for $\mathrm{C}_{21} \mathrm{H}_{30} \mathrm{NOSSi}[\mathrm{M}]+:$ 372.6. Found 372.2.

Experimental for tert-Butyl dimethylphenylsilyl(phenylmethylene)carbamate (32) from $P, P$ diphenyl- $\boldsymbol{N}$-(1-dimethylphenylsilylphenylmethylene)-phosphinic amide: To a $25 \mathrm{~mL}$ Schlenk flask was added compound $7(89 \mathrm{mg}, 0.201 \mathrm{mmol})$ and $2 \mathrm{~mL}$ of THF. The solution was cooled to $0{ }^{\circ} \mathrm{C}$, and conc. $\mathrm{HCl}(1.5 \mathrm{~mL}, 18.1 \mathrm{mmol})$ was added. After the disappearance of starting material (as measured by TLC), $5 \mathrm{~mL}$ of water was added, followed by $\mathrm{K}_{2} \mathrm{CO}_{3}$ (solid) to basify the solution (pH 9). ${ }^{8}$ The solution was then cooled to $0{ }^{\circ} \mathrm{C}$ and $\mathrm{Boc}_{2} \mathrm{O}(53 \mathrm{mg}, 0.24 \mathrm{mmol})$ was added. The reaction was then allowed to warm to room temperature over night. The mixture was taken up with ethyl acetate $(20 \mathrm{~mL})$, washed with water $(1 \times 10 \mathrm{~mL})$, brine $(2 \times 10 \mathrm{~mL})$. The organic layer was dried over anhydrous sodium sulfate, filtered, and concentrated in vacuo. The unpurified reaction mixture was purified using flash chromatography (10\% EtOAc/ hexanes) to yield pure product in $82 \%$ yield over 2 steps.

Experimental for tert-Butyl dimethylphenylsilyl(phenylmethylene)carbamate (32) from $\left(\mathbf{R}_{\mathrm{s}}, \mathbf{R}\right)-\mathrm{N}-(\mathbf{1}-$ dimethylphenylsilylphenylmethylene)-t-butanesulfinamide: To a $25 \mathrm{~mL}$ Schlenk flask was added compound 25 (233 mg, $0.67 \mathrm{mmol})$ and $3 \mathrm{~mL}$ of THF. The solution was cooled to $0{ }^{\circ} \mathrm{C}$, and a $4 \mathrm{~N}$ solution of $\mathrm{HCl}$ in dioxane ${ }^{9}(1.5 \mathrm{~mL}, 6.0 \mathrm{mmol})$ was added. After the disappearance of starting material (as measured by TLC), $2 \mathrm{~mL}$ of water was added, followed by $\mathrm{K}_{2} \mathrm{CO}_{3}$ (solid) to basify the solution (pH 9). ${ }^{6}$ The solution was then cooled to $0{ }^{\circ} \mathrm{C}$ and $\mathrm{Boc}_{2} \mathrm{O}(175 \mathrm{mg}, 0.80 \mathrm{mmol})$ was added. The reaction was then allowed to warm to room temperature over night. The mixture was taken up with ethyl acetate $(20 \mathrm{~mL})$, washed with water $(1 \times 10 \mathrm{~mL})$, brine $(2 \times 10 \mathrm{~mL})$. The organic layer was dried over anhydrous sodium sulfate, filtered, and concentrated in vacuo. The unpurified reaction mixture was purified using flash chromatography (10\% EtOAc/ hexanes) to yield pure product in $77 \%$ yield over 2 steps. 